problems (including mental illness specifically) have communication problems stemming from each of these factors: cortical damage; developmental isolation; and unenlightened educational methods. Psycho-linguistic studies of formal thought disorder in deaf people with schizophrenia (often misdiagnosed in this group) are in progress. The first consultant post in Britain in the psychiatry of deaf children and adolescents has recently been established at Springfield and St George's Hospitals. There must be close links between parents, teachers, educational psychologists and doctors to recognise early and minimise the particular developmental difficulties experienced by deaf children.

Towards the end of the day a panel of researchers emphasised the need for ongoing work in this specialised field.

(a) Contrary to a generally held notion, a community survey appears to have found high levels of unrecognised depressive illness in prelingually deaf adults, using an adaptation of an established two-stage paradigm.

(b) The usefulness of certain phenomenological criteria for the diagnosis of schizophrenia has been re-evaluated. (c) The communication and cultural divide between deaf and hearing people is likely to lead to under-reporting of mental disorder and misdiagnosis.

(d) Questionnaires can help teachers assess the emotional development of their deaf pupils.

The research development manager at the Royal National Institute for the Deaf, Dr Katia Herbst, announced the establishment of a directory of researchers and projects. A plea was made on behalf of those with later onset, acquired deafness, and hard of hearing people.

A decision was taken to set up a small steering party to discuss the constitution of a British Society for Mental Health and Deafness. Such a society would promote British experience in the field and encourage co-ordinated service developments and research.

Note

Proceedings of this conference are available ( $£ 5$ as a text, $£ 10$ on videotape with sign language) from the Deaf Unit at Springfield Hospital, telephone 081-784-2773 (voice) or 081-784-2705 (Minicom).

\title{
Epilepsy and learning disability*
}

\section{T. P. Berney, Consultant Psychiatrist, Prudhoe Hospital, Prudhoe, Northumberland NE42 5NT}

Epilepsy is rife in learning disability and is a major component of any practice: it was chosen as the theme for the annual residential conference of the Mental Handicap Section. Its importance in everyday practice was affirmed by presentations from Ulster and Avon which examined the local prevalence of epilepsy in Down's syndrome. In both, the age of seizure onset had a bimodal distribution, the second peak occurring in the fourth decade. The growth of this group promises an increasing demand on overstretched services.

The scene was set by a series of ten-minute presentations of audit projects which summarised the clinical practice of five services and set some initial criteria for national comparison. There was an adherence to monotherapy, even with the intractable epilepsies; a bias towards carbamazepine and valproate with an avoidance of phenytoin and barbiturates; an annual review including drug levels

* A joint conference between the Section for the Psychiatry of Mental Handicap and the International League against Epilepsy, Jersey, 21-23 November 1991. and liver function; and repeated questioning of the amount and type of medication. The subsequent discussion brought out the difficulty of establishing a reliable fit chart, the lack of an agreed threshold for the use of rectal medication in status epilepticus, and the need to take the overall quality of the patient's life into account. Furthermore the models of audit marked a methodological path for others to follow.

Expert reviews of the modern management of epilepsy filled the following day. The association between epilepsy and psychiatric disturbance was emphasised, of some importance in a field where it is the fashion to replace the psychiatrist with the neurologist (Steven Brown). Immediately relevant to psychiatry was the potential for the psychological control of seizures (Peter Fenwick), and the misdiagnosis of stimulated seizures as epilepsy (Tim Betts). The role of two new drugs was defined further in discussion; lamotrigine for the treatment of myoclonic phenomena and vigabatrin for complex partial seizures. Although at present these are limited to use as an add-on drug in intractable epilepsy, there was optimism for their potential as 'clean' and 
effective primary drugs. For example, the adverse effects of vigabatrin might often represent an incompatability with other drugs, improving their removal. All treatments, including surgery (Charles Polkey), should be judged on their longer-term efficacy over at least five years.

The conference's emphasis on service provision, coupled with the Department's recent choice of 'learning disability', led to a re-examination of the Section's function and title. There was a reluctance to pursue a chameleon fashion which may yet arrive at some politically correct phrase such as 'people facing a developmental challenge'. However, as other services move on, so 'mental handicap' might be left to be seen as isolated, idiosyncratic or, at best, as quaint. There was some favour for 'developmental psychiatry' but this might equally embrace child psychiatry. Like a problematic wine set aside to mature, it was left for time to resolve some of these issues. This debate set the context for the guest lecture, given by Professor Brice Pitt, on the Section's responsibility for public education. This means the development of some enthusiasm for, and skill in working with, the news media together with a readiness to seize the passing opportunity.

The meeting was well-attended by nearly 100 members. It introduced many trainees to the wider world in which they are to seek posts and make their career. Addressing some of the everyday tasks of a mental handicap service, it gave further substance and purpose to a subspecialty which has left behind its earlier self-doubt and recruitment difficulties.

\title{
The Newhamexperience: from hospital into the community during two decades*
}

\author{
David Abrahamson, Consultant Psychiatrist, Goodmayes Hospital, Goodmayes, \\ Ilford, Essex IG3 8XJ
}

The conference presented experience mainly related to de-institutionalisation from Goodmayes Hospital, which it was thought might be particularly relevant at a critical time in the evolution of services.

An initial overview of housing developments emphasised the importance of a varied range of accommodation to suit individual needs and choices. The importance of choice was underlined by studies of long-stay patients' attitudes to leaving hospital which had shown that they were influenced by realistic consideration of what was available in the community. It is facilitated by the several preparation houses which have been developed on the hospital campus.

The value of single houses in the community, both the traditional group home and recent staffed versions, was acknowledged; but not all patients wish to live in such physical and emotional closeness. Several larger projects have been designed to provide more space and privacy with the opportunity for companionship when desired, and are proving very successful.

Other contributions by members of the Newham Rehabilitation Team dealt with the assessment methods used and their results, which have been predominantly encouraging, and with leisure and social activities - a combined group and individual

* Conference on 22 November 1991 at Newham General Hospital, London E16. out-patient clinic patients and a social club which developed from it have been happy experiences.

Investigations of the social networks of hospital and community patients confirmed the support they can give one another: but it is clear that moving 'into the community' does not ensure integration.

The importance of communication and the contribution of pragmatic concepts and techniques was emphasised by another member of the team; one of the few speech and language therapists available to long-term psychiatric patients.

In appraising the morning's contributions, Matt Muijen, Director of Research and Development for Psychiatry, Guy's and St Thomas's Hospitals, pointed out the general success of deinstitutionalisation in terms of quality of life, which throws into focus the lack of comparable facilities for other patients who have not become long-stay, a point which was repeatedly emphasised during the afternoon session. This was in the form of three parallel workshops, on preparation for leaving hospital, community provision and relationships between the agencies concerned, and issues of social and clinical support including modifications of clinical style required in the new settings.

The overall message seemed to be that deinstitutionalism is working but is not yet out of the woods. Concerns included the possibility that the culture of rules and regulations produced by the Registered Homes Act risks turning the clock back. 